muscles were made during extension-flexion movements ( $90^{\circ}$ amplitude) of the elbow joint.

During the stretch phase, patients with PD had notably higher electromyographic activity than did controls; no such differences were observed during release or neutral phases. This finding suggests that rigidity is induced by muscle activity changes, rather than by muscle architecture. The authors also revealed that, in patients with $\mathrm{PD}$, rigidity took 1-2 min to react completely to changes in DBS, which suggests that clinicians should wait at least 2 min before assessing a patient's clinical status following a change in DBS parameters.

Original article Levin J et al. (2008) Objective measurement of muscle rigidity in parkinsonian patients treated with subthalamic stimulation. Mov Disord [doi:10.1002/mds.22291]

\section{A high-dose capsaicin dermal patch provides pain relief in postherpetic neuralgia}

Treatment options are limited for patients with postherpetic neuralgia (PHN), a chronic pain disorder caused by reactivation of the varicella zoster virus. Topical application of capsaicin, an agent used to treat chronic pain syndromes, has been shown to improve PHN-related pain. Backonja et al. have demonstrated that one 60-minute application of a high-dose capsaicin dermal patch provides immediate and long-termreduction in pain in patients with PHN.

This multicenter, double-blind study enrolled 402 patients who had experienced PHN for at least 6 months. Participants were randomly assigned to receive a dermal patch containing a high concentration of capsaicin $\left(640 \mu \mathrm{g} / \mathrm{cm}^{2}\right.$ [8\%]) or a low concentration of capsaicin $\left(3.2 \mu \mathrm{g} / \mathrm{cm}^{2}[0.04 \%]\right)$, which was applied to painful areas for $60 \mathrm{~min}$.

Patients who were treated with the highdose capsaicin patch $(n=206)$ had a significantly greater decrease in pain at day one than did those who received the low-dose patch $(n=196 ; P<0.01)$. The mean pain reduction over 8 weeks and over 12 weeks was almost $10 \%$ more in the high-dose group than in the low-dose group $(P=0.001$ and $P=0.002$, respectively), regardless of whether patients were taking concomitant medications for neuropathic pain. Almost all patients in both treatment groups experienced at least one transient mild to moderate adverse effect as a result of expected capsaicin-related reactions at the application site (e.g. pain, erythema).

The authors conclude that the high-dose capsaicin dermal patch is a safe and effective strategy for sustained pain relief in patients with PHN.

Original article Backonja $\mathrm{M}$ et al. for the NGX-4010 C116 Study Group (2008) NGX-4010, a high-concentration capsaicin patch, for the treatment of postherpetic neuralgia: a randomised, double-blind study. Lancet Neurol 7:1106-1112

\section{Lipophilicity of statins does not alter their protective effect against Alzheimer disease}

Observational studies have suggested that statin use reduces the risk of Alzheimer disease, but the mechanism that underlies this association is unknown. Hypothetically, lipophilic statins could penetrate the bloodbrain barrier, affect cholesterol homeostasis in the brain and influence brain function. Haag et al. conducted a prospective, populationbased cohort study that confirmed a protective effect of statins against Alzheimer disease (AD); however, they also showed that this effect is conferred regardless of the lipophilicity of the drug.

The investigators analyzed data from 6,992 individuals (aged $\geq 55$ years) in The Netherlands who were free of dementia at baseline, had $\geq 6$ months of medication history available during 1991-2005, and had undergone a psychoneurological evaluation (average follow-up 9.2 years). Overall, 582 individuals were diagnosed with AD. Use of either lipophilic statins (simvastatin, atorvastatin or cerivastatin) or hydrophilic statins (pravastatin, fluvastatin or rosuvastatin) was associated with a reduced risk of $A D$ compared with non-use of any cholesterol-lowering drug (odds ratios 0.54 for both). The protective effect was maintained regardless of dosage and duration of statin use and was not influenced by the presence or absence of an APOE-4 allele, which is involved in cholesterol metabolism in the brain.

The authors note that both lipophilic and hydrophilic statins might penetrate the bloodbrain barrier by mechanisms that are not 